\title{
Determination of over current protection thresholds for class $D$ audio amplifiers
}

\author{
Nyboe, Flemming; Risbo, L; Andreani, Pietro
}

Published in:

Proceedings of $23<$ sup $>$ rd $</$ sup $>$ NORCHIP Conference

Link to article, DOI:

10.1109/NORCHP.2005.1597005

Publication date:

2005

Document Version

Publisher's PDF, also known as Version of record

Link back to DTU Orbit

Citation $(A P A)$ :

Nyboe, F., Risbo, L., \& AndreanifP. (2005). Determination of over current protection thresholds for class D audio amplifiers. In Proceedings of 23 NORCHIP Conference (pp. 125-128). [1597005] IEEE.

https://doi.org/10.1109/NORCHP.2005.1597005

\section{General rights}

Copyright and moral rights for the publications made accessible in the public portal are retained by the authors and/or other copyright owners and it is a condition of accessing publications that users recognise and abide by the legal requirements associated with these rights.

- Users may download and print one copy of any publication from the public portal for the purpose of private study or research.

- You may not further distribute the material or use it for any profit-making activity or commercial gain

- You may freely distribute the URL identifying the publication in the public portal 


\title{
Determination of Overcurrent Protection Thresholds for Class D Audio Amplifiers
}

\author{
FLEMMING NYBOE ${ }^{1}$, LARS RISBO ${ }^{2}$ AND PIETRO ANDREANI ${ }^{3}$ \\ ${ }^{1}$ Digital Audio Department, Texas Instruments, Denmark \\ and \\ Orsted $\cdot D T U$, Technical University of Denmark \\ fls (a)ti.com \\ ${ }^{2}$ Digital Audio Department, Texas Instruments, Denmark \\ Iri@ti.com \\ ${ }^{3}$ Orsted $\cdot$ DTU, Technical University of Denmark \\ pa $a$ oersted.dtu.dk
}

\begin{abstract}
:
Monolithic Class-D audio amplifiers typically feature built-in overcurrent protection circuitry that shuts down the amplifier in case of a short circuit on the output speaker terminals. To minimize cost, the threshold at which the device shuts down must be set just above the maximum current that can flow in the loudspeaker during normal operation. The current required is determined by the complex loudspeaker impedance and properties of the music signals played. This work presents a statistical analysis of peak output currents when playing music on typical loudspeakers for home entertainment.
\end{abstract}

\section{Introduction}

A simplified schematic of a class D audio amplifier system is shown in Figure 1. The audio input signal (analog or digital) is converted to a logic-level pulse width modulated (PWM) signal by a modulator (not shown), and level shifted to produce the gate signals for the switches. The two output stage switches are turned on alternately, reproducing the PWM waveform at the switching node $\mathrm{V}_{\mathrm{AMP}}$. The demodulation LC filter then removes the switching frequency components of the PWM signal, leaving only the audio signal on the output node $\mathrm{V}_{\mathrm{SP}}$. The overcurrent protection circuit measures the output current $\mathrm{I}_{\mathrm{AMP}}$ during operation, either by measuring the voltage across the output switch that conducts the current [1], or otherwise.

In a monolithic Class D amplifier design, the output stage switches take up a major fraction of the total die area, and thus the cost of build. Determination of the minimum overcurrent threshold that will not interfere with normal operation, i.e. playing music into the loudspeaker, is necessary to minimize the size of the output switches.

Previous papers have dealt with the topic of finding the maximum possible current in a loudspeaker, caused by any signal limited in magnitude to the supply voltage $V_{B}$ [3], [4]. These papers are from the Class AB amplifier era, and the output current from the amplifier is considered equal to the current in the loudspeaker. As shown in Figure 1, this is not the case for Class D amplifiers, where amplifier output and loudspeaker are separated by a demodulation LC filter. For accurate results, this must be accounted for in computation. Another difference is that while traditional Class $A B$ amplifiers employ high gain feedback loops, causing very low amplifier output impedance, many low-cost Class D amplifiers operate from purely digital input signals, and feedback cannot easily be applied. This causes significant amplifier output impedance, which must also be accounted for in computation.

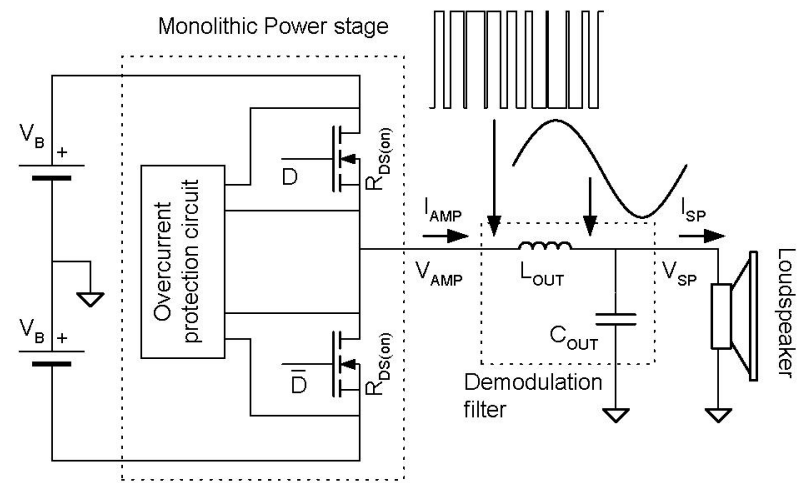

Figure 1: Simplified Class D amplifier system. The input signal is the duty cycle (D) of the gate signals for the switches.

Practical amplifiers can be implemented as shown, or 2 such output stages can be bridge connected to form one Bridge Tied Load (BTL) output stage running from a single $V_{B}$ supply rail. Either way, the maximum output voltage across the loudspeaker is $\pm V_{B}$, and the following results apply.

\section{Computation of peak current}

The maximum possible peak current in a loudspeaker, caused by any signal limited to $\pm V_{B}$ can be derived from the complex impedance $Z_{\mathrm{SP}}(\mathrm{f})$ of the loudspeaker, through the following steps:

1. Measure the complex impedance versus frequency $\mathrm{Z}_{\mathrm{SP}}(\mathrm{f})$ of the loudspeaker 
2. Calculate the complex admittance $Y_{\mathrm{SP}}(\mathrm{f})=1 / Z_{\mathrm{SP}}(\mathrm{f})$

3. Apply an anti-aliasing filter to band-limit $Y_{\mathrm{SP}}(\mathrm{f})$ and avoid discontinuity at the Nyquist frequency [3]

4. Calculate the current impulse response $\mathrm{y}(\mathrm{t})=\operatorname{IFFT}\left(\mathrm{Y}_{\mathrm{SP}}(\mathrm{f})\right)$

5. The maximum current signal is $\mathrm{V}_{\mathrm{B}} \cdot \operatorname{sign}(\mathrm{y}(-\mathrm{t})) * \mathrm{y}(\mathrm{t})$

This procedure is described in [2] and [3] and will not be detailed here. An example of $y(t)$ for a loudspeaker is shown below

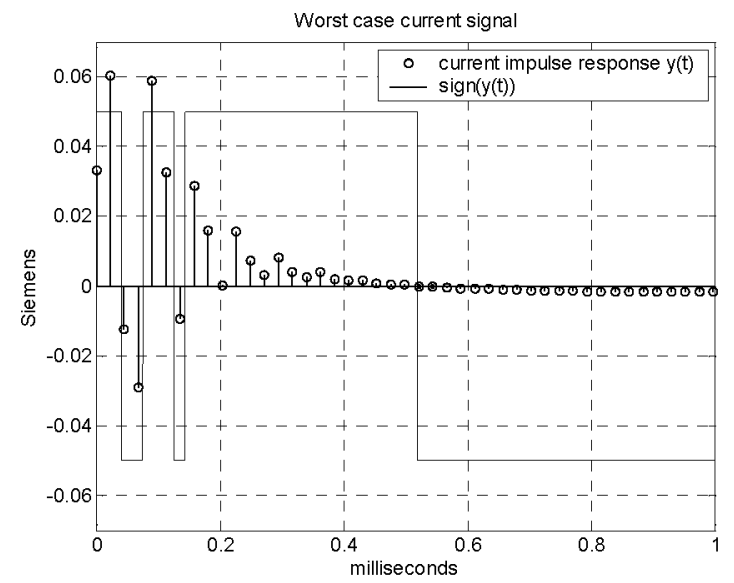

Figure 2: Derivation of the voltage waveform that will produce the maximum possible peak current in a loudspeaker. The amplitude of $\operatorname{sign}(y(t))$ is arbitrarily set to 0.05 to fit in the figure.

$y(t)$ is the current impulse response, i.e. the current waveform the would flow in the loudspeaker in response to an impulse voltage of unit area. The current in the loudspeaker when driven by any voltage waveform $\mathrm{v}(\mathrm{t})$ can be found by convolution of $v(t)$ with the current impulse response, i.e.

$\mathrm{I}_{\mathrm{SP}}(\mathrm{t})=\mathrm{V}_{\mathrm{SP}}(\mathrm{t}) * \mathrm{y}(\mathrm{t})$

$\operatorname{sign}(y(t))$ plotted in Figure 2 illustrates why $\mathrm{V}_{\mathrm{SP}}(\mathbf{t})=\mathrm{V}_{\mathrm{B}} \cdot \operatorname{sign}(\mathrm{y}(-\mathbf{t}))$ is the voltage waveform that will produce the maximum possible convolution integral with $y(t)$, and thus the maximum peak current $I_{S P}(t)$, when applied to the loudspeaker. The time inversion in $y(-t)$ occurs because of the time inversion inherent to convolution.

Since an on-chip overcurrent protection system like the one shown in Figure 1 has no access to the actual loudspeaker current $I_{S P}(t)$, the current limit is instead enforced on the amplifier output current $\mathrm{I}_{\mathrm{AMP}}(\mathrm{t})$. To calculate the maximum possible current $I_{\mathrm{AMP}}(t)$, rather than $I_{S P}(t)$, the calculation must be based not just on $Z_{\mathrm{SP}}(\mathrm{f})$ alone, but on the total impedance of output filter and loudspeaker, as seen from the switching output terminal $\mathrm{V}_{\mathrm{AMP}}$ on the amplifier. Referring to the components in Figure 1, the load impedance at this point is given by
$Z_{A M P}(f)=\frac{Z_{R L} \cdot Z_{S P}+Z_{R L} \cdot Z_{C}+Z_{S P} \cdot Z_{C}}{Z_{C}}$

where

$$
\begin{aligned}
Z_{C} & =\frac{1}{i 2 \pi \cdot f \cdot C_{\text {OUT }}} \\
Z_{R L} & =R_{\text {OUT }}+i 2 \pi \cdot f \cdot L_{\text {OUT }} \\
Z_{S P} & =Z_{S P}(f)
\end{aligned}
$$

and $\mathrm{R}_{\mathrm{OUT}}$ is the total output impedance of the amplifier at the loudspeaker terminals.

Since one of the output stage switches is turned on at any point in time, the on-resistance $\mathrm{R}_{\mathrm{DS}(\mathrm{on})}$ will act exactly like series resistance in the output inductor $\mathrm{L}_{\mathrm{OUT}}$ and $R_{\text {OUT }}$ is the sum of $R_{D S(\text { on })}$ and the actual inductor series resistance $\mathrm{R}_{\mathrm{LOUT}}$. For BTL configurations, each half of the output stage has its own output LC filter and contribution to output resistance. Since the two output filter capacitors, as seen from the loudspeaker terminals, are series connected, we get

$$
\begin{aligned}
& R_{\text {OUT }}=2 \cdot R_{D S(o n)}+2 \cdot R_{\text {LOUT,BTL }} \\
& L_{\text {OUT }}=2 \cdot L_{\text {OUT }, B T L} \\
& C_{\text {OUT }}=\frac{1}{2} C_{\text {OUT }, B T L}
\end{aligned}
$$

to be used in equation (2) for a BTL power stage.

\section{Calculated vs. Measured maximum currents}

As indicated in Figure 2, $y(t)$ is only defined in discrete time, due to the finite frequency range of the impedance measurement $Z_{\mathrm{SP}}(\mathrm{f})$, from which it is found. The sampling interval is determined by the frequency range of $Z_{\mathrm{SP}}(\mathrm{f})$, and if the measurement frequency limit is set to $22050 \mathrm{~Hz}$, the sampling frequency will be $44100 \mathrm{~Hz}$, which is the sampling frequency of audio CDs. The worst case current excitation signal sign(y(-t)) can then be written onto an audio $\mathrm{CD}$ at maximum signal level, and played on the loudspeaker to verify the worst case current by measurement. This has been done for 4 different loudspeakers, and the results are shown in Figure 3.

The loudspeakers selected are not particularly high-end, but represent those typically shipped with mediumpowered home theatre solutions, since this is the primary market space for monolithic Class D amplifiers.

The checkered bars are measured peak currents when playing the $\operatorname{sign}(\mathrm{y}(-\mathrm{t}))$ functions from the $\mathrm{CD}$ on each respective loudspeaker. The amplifier used was a BTL Class $\mathrm{D}$ amplifier without feedback, and with total $\mathrm{R}_{\mathrm{OUT}}$ of $0.50 \mathrm{ohms}$. The measured current has been normalized by the supply voltage $V_{B}$.

The calculated maximum currents are found by use of equation (1), where $y(t)$ in the case of the verticallystriped bars is based just on the measured 
$Z_{\mathrm{SP}}(\mathrm{f})+0.50 \mathrm{ohm}$, i.e. the amplifier output resistance is accounted for, but the LC output filter is not. These results are seen to be very inaccurate, more that a factor of two in case of the Panasonic Satellite loudspeaker. The non-systematic nature of the errors means the results can not be used even as best- or worst-case estimates. For the horizontally-striped bars, the calculation of $y(t)$ is based on $Z_{\mathrm{AMP}}(\mathrm{f})$ as given by equation (2), thereby accounting for both the output resistance and LC filter components. With the exception of the Kenwood loudspeaker, these results are more accurate, illustrating the importance of including the LC filter in the calculations.

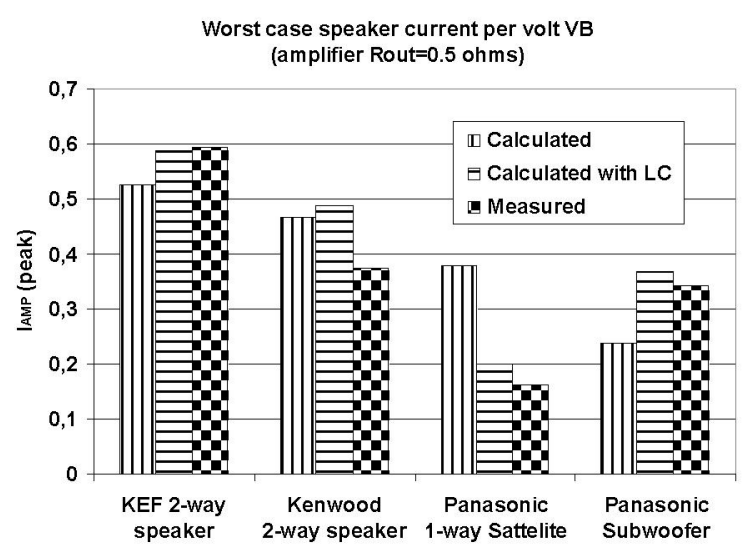

Figure 3: Calculated vs. measured worst case currents for 4 loudspeakers

The remaining inaccuracies are believed to be related to the fact that the calculated results are derived from an $Z_{\mathrm{SP}}(\mathrm{f})$, which is in fact only a small signal model of the loudspeaker. During measurement of the worst case currents, the loudspeaker membranes had quite large excursions, even though the amplifier supply voltage was kept at a moderate level of $V_{B}=3 \mathrm{~V}$. Using even lower $V_{B}$ voltages might have resulted in better agreement between calculation and measurement. This deserves a more detailed study, since the final interest obviously is the level of peak currents that occur when amplifiers operate at realistic supply voltages.

\section{Statistical analysis with music signals}

The currents measured and calculated above are very large compared to the nominal impedances of the loudspeakers. The KEF 2-way speaker (KEF KHT2005.2) is labeled 8 ohms, but its worst case peak current is almost $0.6 \mathrm{~A}$ per volt $\mathrm{V}_{\mathrm{B}}$.

For low cost audio amplifiers it may not always realistic to design the output stage to deliver the current needed under absolute worst case conditions. Such amplifiers can instead be designed so that in case of an overcurrent detection, they only shut down the output stage briefly, and then restart it, rather than shutting down the amplifier completely. If implemented correctly, the resulting drop-outs can be almost inaudible, given that they are brief and occur rarely.

This calls for an analysis of music signals, to determine how likely it is for a typical music signal to cause a given peak current level in a loudspeaker load. To answer this, a selection of $32 \mathrm{CD}$ music tracks from varied genres has been analyzed. Using $\mathrm{y}(\mathrm{t})$ for the KEF 2-way loudspeaker, the entire current waveform $I_{\mathrm{SP}}(t)$ resulting from playing each music track at full volume ${ }^{1}$ into the loudspeaker has been calculated by use of equation (1). The peak current for each track can then be found from each calculated waveform. The 32 results so obtained had a mean value of $\mu=0.196 \mathrm{~A}$ and a standard deviation of $\sigma=0.049 \mathrm{~A}$. Based on these numbers, the probability that a randomly selected audio track will produce a peak current that exceeds a current I is given by:

$P\left\{I_{S P, P E A K}>I\right\}=1-\Phi\{I\}$

where $\Phi(\mathrm{I})$ is the cumulative normal distribution with mean $\mu$ and standard deviation $\sigma$.

$\mathrm{P}\left\{\mathrm{I}_{\mathrm{SP}, \mathrm{PEAK}}>\mathrm{I}\right\}$ is graphed in Figure 4. The figure should be read as follows: The curve "Music, gain=0 dB" is at $47 \%$ for $0.20 \mathrm{~A}$. This means that based on the distribution of peak currents from the 32 music tracks analyzed, $47 \%$ of randomly selected tracks will produce an output current peak of $0.20 \mathrm{~A} / \mathrm{V}_{\mathrm{B}}$ or more, when played at full volume into the KEF 2-way loudspeaker. The vertical lines show similar results for load resistors of selected values. Here there is no variation in current since for a resistive load $R$, any output signal with a peak voltage of $\pm V_{B}$ will produce a peak current of $\pm V_{B} / R$. Taking $R_{\text {out }}$ into account, all 32 audio tracks would produce a current of $\mathrm{V}_{\mathrm{B}} /(8+0.5)=0.12 \mathrm{~A} / \mathrm{V}_{\mathrm{B}}$ into an $8 \mathrm{ohm}$ resistor, as indicated by the line "Nominal imp ( 8 ohms)". This shows that $94 \%$ of randomly selected audio tracks will produce a larger peak current into the KEF 2-way speaker than into an $8 \mathrm{ohm}$ resistor. Similarly, 15\% of randomly selected tracks will produce larger peak current than a load resistor with the same value as the minimum impedance of the loudspeaker, which is $3.3 \mathrm{ohms}$ at $350 \mathrm{~Hz}$. The "Worst case" line indicates the maximum possible current from Figure 3.

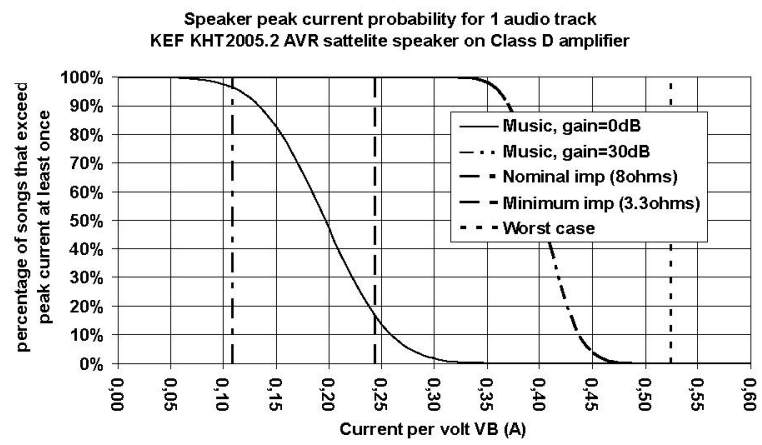

Figure 4: Statistical distribution of loudspeaker peak current, playing one $\mathrm{CD}$ track

The results so far are based on playing music at full volume, but without overdrive (clipping). Most amplifier

\footnotetext{
${ }^{1}$ Full volume is defined such that an all-zero digital code on the CD produces a no-load amplifier output voltage of $-\mathrm{V}_{\mathrm{B}} \mathrm{V}$, and an all-one digital code $+\mathrm{V}_{\mathrm{B}} \mathrm{V}$.
} 
products have the feature of applying gain to the audio input signal (analog or digital), with signal clipping as a result. In some end user products as much as $30 \mathrm{~dB}$ gain can be applied to a digital input signal which may already utilize the full digital headroom. Though this results in severe distortion and very low audio fidelity, the situation is technically within normal operation of the amplifier, and must be accounted for in design.

Calculation of peak currents that occur when playing $30 \mathrm{~dB}$ overdriven audio into the KEF 2-way speaker has been made simply by applying $30 \mathrm{~dB}$ gain to the 32 audio tracks (still constrained by the range of the digital code), and then convoluting the result by the current impulse response $y(t)$. The resulting distribution of peak currents is shown by the curve "Music, gain=30dB" in Figure 4. The peak currents are now much higher, which is not surprising from the intuitive point of view that a heavily clipped audio signal bears stronger resemblance with the worst-case signal shown in Figure 2, than an unclipped signal does.

For designs where a very low rate of system drop-outs is required, the probability that a given output current will be exceeded at least once per $n$ (rather than 1) audio tracks can be found simply by

$$
P_{n}\left\{I_{S P, P E A K}>I\right\}=1-\Phi\{I\}^{n}
$$

where $\Phi(\mathrm{I})$ is the cumulative normal distribution with mean $\mu$ and standard deviation $\sigma$.

This probability function is plotted below for $n=100$ and $0 \mathrm{~dB}$ vs. $30 \mathrm{~dB}$ gain, based on the same data as Figure 4 .

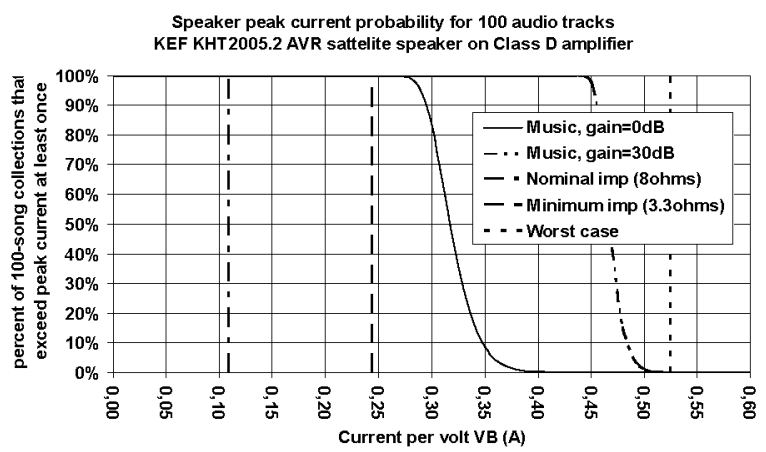

Figure 5: Statistical distribution of peak current, playing $100 \mathrm{CD}$ tracks

It is seen that when playing back 100 audio tracks into this loudspeaker, the probability that current will at some point exceed that of a load resistor with the same value as the minimum loudspeaker impedance (3.3 ohms) driven by the same signal, is almost 1 . This contradicts measured results in [4], where this is claimed not to have happened for hundreds of hours of music played on 7 different loudspeakers.

It is also seen that the probability of the output current exceeding $0.4 \mathrm{~A} / \mathrm{V}_{\mathrm{B}}$ is very small (unless the signal is clipped). Setting the overcurrent threshold at this level would thus result in very infrequent drop-outs. For a supply voltage of $\mathrm{V}_{\mathrm{B}}=30 \mathrm{~V}$, it corresponds to $30 \cdot 0.4=12 \mathrm{~A}$
The above analysis can readily be applied to any given amplifier with any given loudspeaker. This is useful for systems where the loudspeakers are sold bundled with the amplifier, as is common for lower-power amplifiers that employ monolithic Class-D output stages. For higher power solutions, where amplifier and loudspeakers are typically sold separately, a number of loudspeakers that represent those typically used with an amplifier can be analyzed. It is worth noting that higher power multidriver loudspeakers will tend to cause higher peak currents (even for the same nominal impedance) due to the higher complexity of the crossover networks [3].

\section{Summary}

A method for calculating maximum peak currents for given combinations of amplifiers and loudspeakers has been presented. The basic approach has been described in earlier papers, but the effects of amplifier output resistance and the LC output filters used in Class D amplifiers have been included in calculations here. It has been shown that these additions are necessary to obtain results of useful accuracy for Class D amplifier systems. Since it is not always realistic to design low cost amplifier systems for the absolute worst case current, a statistical analysis of peak currents during normal music playing has been added. The results for a single loudspeaker chosen for this analysis contradict those of [4], since it is shown that the output current will frequently exceed that of a resistor with the same value as the minimum impedance of the loudspeaker, driven by the same signal.

Finally, it is shown that overdriving the audio signal increases the peak currents significantly.

\section{References}

[1] Circuit for Amplifying and Outputting Audio Signals Masashi Oki, Kazuhiro Okamoto. U.S. Patent no. 6,469,575 B2 (2002)

[2] Peak Transient Current and Power Into a Complex Impedance

Preis, D. and Schroeter, J

AES preprint \#2337, 80 ${ }^{\text {th }}$ Convention (1986)

[3] Computing Peak Currents Into Loudspeakers Lipshitz, Stanley P. \& Vanderkooey, J. AES preprint \#2411,81st Convention (1986)

[4] Audio Power Amplifiers for Loudspeaker Loads Benjamin, Eric AES preprint \#3432, $93^{\text {rd }}$ Convention (1992) 